\title{
Plaque Forming Units per Animal
}

National Cancer Institute

\section{Source}

National Cancer Institute. Plaque Forming Units per Animal. NCI Thesaurus. Code

C122221.

A unit of measure expressed in plaque forming unit(s) per animal. 